\title{
MicroRNA-21-5p promotes epithelial to mesenchymal transition by targeting SRY-box 17 in endometrial cancer
}

\author{
CUILAN WANG, QING LI and YUAN HE \\ Department of Gynecology and Obstetrics, Jinan Maternity and Child Health Care Hospital, \\ Jinan, Shandong 250001, P.R. China
}

Received April 14, 2018; Accepted July 24, 2018

DOI: $10.3892 /$ or.2020.7556

\begin{abstract}
MicroRNA (miRNA/miR)-21-5p has been proposed as an oncogenic miRNA in human tumors; however, the exact role of miR-21-5p has not been fully determined in endometrial cancer. SRY-box 17 (SOX17) is associated with endometrial cancer development and progression; however, the regulatory mechanisms underlying SOX17 expression in endometrial cancer remain unclear. In the present study, tumor samples were collected from 160 postmenopausal women with endometrial cancer. All tumor samples were examined for miR-21-5p expression by reverse transcription-quantitative polymerase chain reaction (RT-qPCR). The results demonstrated that miR-21-5p expression was associated with shorter overall survival. In addition, overexpression of miR-21-5p promoted epithelial to mesenchymal transition (EMT), whereas silencing miR-21-5p reversed EMT in endometrial cancer cell lines. Using RT-qPCR and western blotting, it was revealed that overexpressing miR-21-5p significantly inhibited SOX17 protein expression in endometrial cancer cell lines. Furthermore, as determined by luciferase reporter assay, ectopic expression of miR-21-5p inhibited the activity of the SOX17 mRNA 3'-untranslated region (3'UTR), whereas silencing miR-21-5p promoted the activity of the SOX17 mRNA 3'UTR in endometrial cancer cell lines. Overexpression of SOX17 promoted mesenchymal to epithelial transition, whereas silencing SOX17 induced EMT in endometrial cancer cell lines. In addition, tumor SOX17 expression was associated with better overall survival. Therefore, it may be concluded that miR-21-5p promotes EMT by targeting SOX17 in human endometrial cancer.
\end{abstract}

Correspondence to: Dr Yuan He, Department of Gynecology and Obstetrics, Jinan Maternity and Child Health Care hospital, 3 Jianguo Xiaojing Road, Jinan, Shandong 250001, P.R. China E-mail: heyuanjnmch@163.com

Key words: microRNA-21-5p, SRY-box 17, epithelial to mesenchymal transition, mesenchymal to epithelial transition, endometrial cancer

\section{Introduction}

Endometrial cancer is the most common gynecological cancer in developed countries (1), and comprises a range of diseases with distinct genetic and molecular features (2). Identification of novel molecular targets may improve classification and treatment of endometrial cancer.

SRY-box 17 (SOX17) encodes a 414-amino acid protein member of the SRY-related HMG-box (SOX) transcription factor superfamily (3). Dysregulation of SOX17 serves an important role in the development and progression of numerous types of cancer, including breast cancer (4), gastric cancer (5), lung cancer (6), esophageal cancer (7) and endometrial cancer (8). Low SOX17 protein expression is correlated with poor prognosis in patients with cancer (9). Conversely, SOX17 expression is correlated with longer overall survival and it has been reported to increase sensitivity to cisplatin in endometrial cancer (10). Therefore, SOX17 has been proposed as a tumor suppressor in endometrial cancer; however, the regulatory mechanisms underlying SOX17 expression remain unclear in endometrial cancer.

Epithelial to mesenchymal transition (EMT) is an important process that leads to cancer metastasis, which is characterized by loss of epithelial markers, such as E-cadherin, and an increase in mesenchymal markers, such as fibronectin (11). MicroRNAs (miRNAs/miRs) represent a large class of small ( $22 \mathrm{nt})$ RNA molecules, that are important post-transcriptional regulators (12). miRNAs can regulate the expression of key target genes, such as SOX17, which are involved in metastasis of endometrial cancer $(13,14)$. Numerous miRNAs are dysregulated in endometrial cancer (15) and participate in EMT (16). For example, miR-21-5p has been reported to promote the progression of endometrial cancer (17). However, the mechanisms underlying the effects of miR-21-5p on progression of endometrial cancer remain unclear. The present study aimed to investigate the roles of miR-21-5p and SOX17 in EMT in endometrial cancer.

\section{Materials and methods}

Endometrial cancer samples. The present study was approved by the Ethics Committee of Jinan Maternity and Child Health Care Hospital (Jinan, China), and each patient provided written informed consent at the time of enrollment. A total of 
160 postmenopausal women diagnosed with primary endometrial cancer were recruited between January 2003 and January 2006 at Jinan Maternity and Child Health Care Hospital (Jinan), China). All patients had not been treated with preoperative chemotherapy or radiation. The study population had a median age of 60.5 years (range, 50.0-74.0 years) with a median body mass index of $23.3 \mathrm{~kg} / \mathrm{m}^{2}$ (range, $20.4-38.3 \mathrm{~kg} / \mathrm{m}^{2}$ ). Tumor samples were obtained during surgery following removal of the necessary amount of endometrial cancer tissue for routine pathological examination. The corresponding adjacent normal tissue sample was selected $>3 \mathrm{~cm}$ away from the site at which the primary tumor was sampled. Two pathologists reviewed all tumor tissues and adjacent normal tissues. All tissue specimens were snap-frozen in liquid nitrogen within $1 \mathrm{~h}$ of removal and were stored at $-80^{\circ} \mathrm{C}$.

Cell culture. The HEC-1A, HEC-1B, RL95-2 and AN3CA human endometrial cancer cell lines were purchased from the America Type Culture Collection. The cell lines were maintained in Eagle's Minimum Essential Medium (Gibco; Thermo Fisher Scientific, Inc.) supplemented with $10 \%$ heat-inactivated ( $56^{\circ} \mathrm{C}, 30 \mathrm{~min}$ ) fetal bovine serum (FBS; Gibco; Thermo Fisher Scientific, Inc.). Monolayer cultures were incubated at $37^{\circ} \mathrm{C}$ in a $95 \%$ humidified atmosphere containing $5 \% \mathrm{CO}_{2}$. Cellular morphology was observed using a light microscope (Leica Microsystems, Inc.).

SOX17 plasmids. SOX17-expressing plasmids and empty vectors (mock, pcDNA 3.1), and short hairpin RNA (sh)SOX17 and a scramble control were purchased from Tiangen Biotech Co., Ltd. Human SOX17 was cloned by reverse transcription-PCR (RT-PCR) from cDNA derived from adjacent normal tissue samples of patients with endometrial cancer (forward, 5'-GTTCGGATCCGCCATGAGCAGCCC GGATGCG-3'; reverse, 5'-ATGTGAATTCCACGTCAG GATAGTTGCAGTA-3'), and its sequence was confirmed by direct sequencing of PCR products. Sequencing analysis was carried out using the BigDye Terminator v1.1 sequencing kit, according to manufacturer's protocol (Applied Biosystems; Thermo Fisher Scientific, Inc.). To generate human SOX17 expression constructs, the entire encoding region of its cDNA was subcloned in frame into pcDNA 3.1 (Invitrogen; Thermo Fisher Scientific, Inc.). Suppression of SOX17 expression was induced using shSOX17: 5'-CGCACGGAAUUCGAACAG UAU-3'. Negative control shRNA (5'-ACCGAGCAGUACAAC GGGAAC-3'; Applied Biosystems; Thermo Fisher Scientific, Inc.) was used as a control.

miR-21-5p sequences. Pre-miR-21-5p and a control miR, and anti-miR-21-5p and a scramble miR control were purchased from Ambion; Thermo Fisher Scientific, Inc. The sequences were as follows: Pre-miR-27a-3p, 5'-AACAUCAGUCUGAUA AGCUAUU-3'; control miR, 5'-AACCAUUUGAGAGUC AUCAAGA-3'; anti-miR-21-5p, 5'-UCAACAUCAGUCUGA UAAGCUA-3'; scramble miR control, 5'-CAGUACUUUUGU GUAGUACAA-3'.

Transfection. Cell transfection was performed as described previously (18). For transfection experiments, HEC-1A and AN3CA cells were cultured in serum-free medium without antibiotics at $60 \%$ confluence for $24 \mathrm{~h}$, and were then transfected with $0.5 \mu \mathrm{g}$ SOX17-expressing plasmids or empty vectors, or $10 \mathrm{nM}$ pre-miR-21-5p or control miR using FuGENE ${ }^{\circledR}$ HD transfection reagent (Roche Diagnostics), according to the manufacturer's protocol.

After incubation for $6 \mathrm{~h}$, the medium was removed and replaced with normal culture medium (serum-free medium without antibiotics) for $24 \mathrm{~h}$. Subsequently, the MTT assay, RT-qPCR, RT-quantitative PCR (RT-qPCR), western blotting, immunocytochemistry and luciferase reporter assay were performed.

$R T$-qPCR for mRNA detection. The analysis of mRNA via RT-qPCR was performed as described previously $(18,19)$. Briefly, total cellular RNA was extracted from cultured cells using TRIzol ${ }^{\circledR}$ reagent (Invitrogen; Thermo Fisher Scientific, Inc.) and $2 \mu \mathrm{g}$ total RNA was reverse transcribed using M-MLV reverse transcriptase (Promega Corporation), according to the manufacturer's protocol. The PCR thermocycling conditions were as follows: Denaturation for $30 \mathrm{sec}$ at $95^{\circ} \mathrm{C}$, followed by annealing for $45 \mathrm{sec}$ at $52-58^{\circ} \mathrm{C}$ depending on the primers used, and extension for $45 \mathrm{sec}$ at $72^{\circ} \mathrm{C}$. Each PCR reaction was performed for 28-32 cycles. RT-qPCR was performed using a StepOne ${ }^{\mathrm{TM}}$ real-time PCR system (Applied Biosystems; Thermo Fisher Scientific, Inc.) and Fast SYBR Green Master Mix (Applied Biosystems; Thermo Fisher Scientific, Inc.). Data are shown as relative expression levels after normalization to GAPDH. The following primers were used: Fibronectin, forward 5'-TTTTGACAACGGGAAGCA TTATCAGATAA-3', reverse 5'-TGATCAAAACATTTCTCA GCTATTGG-3'; N-cadherin, forward 5'-CACTGCTCAGGA CCCAGAT-3', reverse 5'-TAAGCCGAGTGATGGTCC-3'; vimentin, forward 5'-CGGGATCCCGCCCTCGTTCGCCTC TTCTC-3', reverse 5'-CGGAATTCCGATATCGCCTGCCAC TGAGTG-3'; E-cadherin, forward 5'-TCAACGATCCTGACC AGCAGTTCG-3', reverse 5'-GGTGAACCATCATCTGTG GCGATG-3'; and GAPDH, forward 5'-CGGAGTCAACGG ATTTGGTCGTAT-3' and reverse 5'-AGCCTTCTCCATGGT GGTGAAGAC-3. Quantification was performed using the $2^{-\Delta \Delta \mathrm{Cq}}$ method (20).

$R T$-qPCR for miRNA detection. The analysis of miRNA expression was performed using RT-qPCR. Total RNA was isolated from cells or tissues using the mirVana ${ }^{\mathrm{TM}}$ miRNA Isolation kit (cat. no. AM1561; Ambion; Thermo Fisher Scientific, Inc.). The detection of mature form of miRNAs was performed using the mirVana ${ }^{\mathrm{TM}}$ RT-qPCR miRNA Detection kit (SYBR-Green) and RT-qPCR Primer Sets (Ambion; Thermo Fisher Scientific, Inc.), according to the manufacturer's instructions. The thermocycling conditions were as follows: One cycle at $50^{\circ} \mathrm{C}$ for $2 \mathrm{~min}$, one cycle at $95^{\circ} \mathrm{C}$ $10 \mathrm{~min}$, followed by 40 cycles at $95^{\circ} \mathrm{C}$ for $15 \mathrm{sec}$ and $60^{\circ} \mathrm{C}$ for $30 \mathrm{sec}$, for extension. The following primer sequences were used: miR-21-5p, forward 5'-TCGCTCGAGATTTTTTTT TATCAAGAGGG-3', reverse 5'-TCGGCGGCCGCGACA AGAATGAGACTTTAATC-3'; U6, forward 5'-GCTTCG GCAGCACATATACTAAAAT-3', reverse 5'-CGCTTCACG AATTTGCGTGTCAT-3'. The U6 small nuclear RNA was used as an internal control. Quantification was performed using the $2^{-\Delta \Delta \mathrm{Cq}}$ method (20). 
Western blot analysis. Western blot analysis was performed as described previously (18). Total protein was prepared using extraction buffer comprising $\mathrm{NaCl} / \mathrm{P}_{\mathrm{i}}$ supplemented with $0.5 \%$ Triton $\mathrm{X}-100,1 \mathrm{mM}$ EDTA, $1 \mathrm{mM}$ phenylmethyl sulfonyl fluoride and complete protease inhibitors (Roche Diagnostics). The concentration of each protein lysate was determined using a bicinchoninic acid protein assay kit (Thermo Fisher Scientific, Inc.). Equal amounts of total protein $(50 \mu \mathrm{g} /$ lane $)$ were separated by $12 \%$ SDS/PAGE. Subsequently, samples were transferred to nitrocellulose membranes and blocked for $60 \mathrm{~min}$ at room temperature in $\mathrm{NaCl} / \mathrm{P}_{\mathrm{i}}$ containing $5 \%$ skim milk powder $(\mathrm{w} / \mathrm{v})$. The membranes were immunoblotted using the following primary antibodies: Anti-fibronectin (cat. no. ab2413; 1:500; Abcam), anti-N-cadherin (cat. no. ab18203; 1:500; Abcam), anti-vimentin (cat. no. ab92547; 1:500; Abcam), anti-E-cadherin (cat. no.ab40772; 1:500; Abcam), anti-SOX17 (cat. no. ab224637; $1: 500 ;$ Abcam) and anti- $\beta$-actin (cat.no.ab8227 1:500; Abcam) overnight at $4^{\circ} \mathrm{C}$. Subsequently, they were incubated with IRDye-800-conjugated anti-rabbit secondary antibodies (cat. no. ab6940, 1:10,000; Abcam) for $30 \mathrm{~min}$ at room temperature. The specific proteins were visualized using the Odyssey ${ }^{\mathrm{TM}}$ Infrared Imaging system (LI-COR Biosciences). $\beta$-actin expression was used as an internal control to confirm equal loading of the protein samples.

MTT assay. The MTT assay was performed as described previously (18). The effects of pre-miR-21-5p, control miR, anti-miR-21-5p, scramble miR control, SOX17-expressing plasmids, empty vectors, shSOX17 and scramble control on the proliferation of endometrial cancer cell lines was assessed by MTT assay (Sigma-Aldrich; Merck KGaA). Briefly, cells were plated in 96-well plates at a density of $8 \times 10^{3}$ cells/well in Eagle's Minimum Essential Medium containing 10\% FBS at $37^{\circ} \mathrm{C}$ in an incubator containing $5 \% \mathrm{CO}_{2}$ for $12 \mathrm{~h}$. Cells were transfected for $48 \mathrm{~h}$. Subsequently, MTT $(5 \mathrm{mg} / \mathrm{ml})$ was added to the wells $(20 \mu \mathrm{l} /$ per well $)$. The plates were then incubated for $4 \mathrm{~h}$ at $37^{\circ} \mathrm{C}$, the supernatant was removed and $150 \mu \mathrm{l}$ dimethyl sulfoxide was added to each well for $10 \mathrm{~min}$. The absorbance of each well was then measured using a

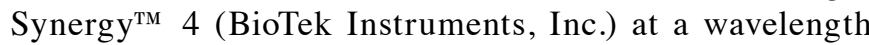
of $570 \mathrm{~nm}$, with the reference wavelength set at $630 \mathrm{~nm}$. Absorbance was directly proportional to the number of surviving cells.

Bioinformatics analysis. Bioinformatics analysis was performed as described previously (21). miRanda (August 2010 Release; http://www.microrna.org/microrna/home.do) was used to identify the target genes of miR-21-5p.

Luciferase reporter assay. Luciferase reporter plasmids containing the wild type SOX17 mRNA 3' untranslated region (3'UTR) and mutant SOX17 mRNA 3'UTR were obtained from Tiangen Biotech, Co. Ltd. The luciferase reporter assay was performed as described previously (21). For reporter assays, $1 \times 10^{6}$ cells were transiently transfected with reporter plasmids $(0.1 \mu \mathrm{g})$ and $10 \mathrm{nM}$ pre-miR-21-5p or control miR using Lipofectamine ${ }^{\circledR} 2000$ (Invitrogen; Thermo Fisher Scientific, Inc.). Reporter assays were performed $36 \mathrm{~h}$ post-transfection using the Dual-Luciferase ${ }^{\circledR}$ Reporter Assay system (Promega Corporation), normalized to Renilla luciferase.

Immunohistochemistry. Immunohistochemistry was performed as described previously (22). Samples were incubated with anti-SOX17. (cat. no. ab224637; 1:500; Abcam) for $12 \mathrm{~h}$ at $4^{\circ} \mathrm{C}$. Subsequently, samples were incubated with IRDye-800-conjugated anti-rabbit secondary antibodies (cat. no. ab6940; 1:10,000; Abcam) for $30 \mathrm{~min}$ at room temperature. Samples were observed under a light microscope. Slides were assessed by quantitative image analysis using the Aperio Image Analysis toolbox (Leica Biosystems, Inc.). Staining intensity and percentage of positive nuclei were recorded after manually segmenting the tumor from adjacent stroma. SOX17 expression levels were semi-quantitatively classified based on the total scores of percentage positivity of stained tumour cells and staining intensity. Namely, the percentage positivity was scored as ' 0 ' if $<5 \%$ (negative), ' 1 ' if $5-30 \%$ (sporadic), ' 2 ' if $30-70 \%$ (focal) and ' 3 ' if $>70 \%$ (diffuse) of cells were stained, whereas staining intensity was scored relative to known positive and negative controls as ' 0 ' if no staining, ' 1 ' if weakly stained, ' 2 ' if moderately stained (intermediate level between strong and weak) and ' 3 ' if strongly stained. The final SOX17 expression score was defined as follows; 'SOX17-' if the sum of the percentage positivity score and the staining intensity score was 0-1, 'SOX17 1+' if the sum was 2-3, 'SOX17 2+' if the sum was 4-5 and 'SOX17 3+' if the sum was 6. SOX17and SOX17 1+ were defined as low expression. SOX17 2+ and SOX17 3+ were defined as high expression.

Statistical analysis. The results were analyzed using SAS software (version 9.4; SAS Institute, Inc.). Data are presented as the means \pm standard error of the mean of separate experiments $(n=3)$. Samples were analyzed by two-tailed Student's t-test for the comparison of two groups, unless otherwise indicated. $\chi^{2}$ tests were used for comparison of categorical variables. The correlation between miR-21-5p and SOX17 expression was analyzed by Spearman correlation (23). Overall survival was analyzed by Kaplan-Meier methods $(24,25)$. Survival was compared in terms of miR-21-5p and SOX17 expression by Kaplan-Meier analysis and log-rank test (two-tailed). $\mathrm{P}<0.05$ was considered to indicate a statistically significant difference.

\section{Results}

Association between miR-21-5p expression and the clinicopathological features of endometrial cancer. Normal endometrial samples were used as controls to determine relative miR-21-5p expression in endometrial cancer tissues. If the relative value of $\mathrm{miR}-21-5 \mathrm{p}$ expression was $\geq 1$, it was defined as high expression. If the relative value of miR-21-5p expression was $<1$, it was defined as low expression. The association between miR-21-5p expression and clinicopathological features is summarized in Table I. The results demonstrated that miR-21-5p expression was associated with lymphatic metastasis $(\mathrm{P}<0.05)$.

Association between miR-21-5p expression and overall survival. To detect miR-21-5p expression in endometrial cancer tissues and adjacent normal tissues, miRNA was isolated from 
Table I. Association between miR-21-5p expression and the pathological parameters of endometrial cancer.

\begin{tabular}{lrrr}
\hline & \multicolumn{2}{c}{$\begin{array}{c}\text { miR-21-5p } \\
\text { expression }\end{array}$} & \\
\cline { 2 - 3 } & Low (\%) & High (\%) & P-value \\
\hline Clinical parameter & $53(40)$ & $79(60)$ & $<0.05$ \\
$\quad$ No & $6(21)$ & $22(79)$ & \\
$\quad$ Yes & & & \\
Invasion depth & $40(39)$ & $63(61)$ & 0.06 \\
$<1 / 2$ & $13(34)$ & $25(66)$ & \\
$>1 / 2$ & $6(32)$ & $13(68)$ & \\
Limited to endometrium & &
\end{tabular}

P-values were calculated using $\chi^{2}$ tests for categorical variables. Percentages show the ratios of patients with high miR-21-5p or low miR-21-5p expression for each item. miR-21-5p, microRNA-21-5p.

160 pairs of adjacent normal tissues and endometrial cancer tissues. RT-qPCR was then performed. The results indicated that miR-21-5p expression was not significantly altered in endometrial cancer tissues compared with in normal tissues (Fig. 1A). Kaplan-Meier curves were applied to evaluate overall survival of the 160 patients with primary endometrial cancers, stratified based on tumor expression of miR-21-5p. There was a significant difference between the two overall survival curves; survival among patients with high miR-21-5p expression was much poorer than survival among patients with low miR-21-5p expression (Fig. 1B).

Overexpression of miR-21-5p promotes EMT in endometrial cancer cell lines. To examine miR-21-5p expression in HEC-1A, HEC-1B, RL95-2 and AN3CA endometrial cancer cell lines, RT-qPCR was conducted. miR-21-5p expression was highest in AN3CA cells and was lowest in HEC-1A cells (Fig. 2A). HEC-1A cells were selected to study the overexpression of miR-21-5p, since endogenous miR-21-5p was very low in this cell line. AN3CA cells were selected to study miR-21-5p knockdown, since endogenous miR-21-5p was very high in this cell line. To determine whether miR-21-5p can promote EMT, HEC-1A cells were transfected with control miR (mock) or pre-miR-21-5p. The results demonstrated that miR-21-5p expression was increased by pre-miR-21-5p in HEC-1A cells (Fig. 2B); this increase in miR-21-5p induced visible alterations in HEC-1A cell morphology (Fig. 2C). To confirm that these alterations were associated with EMT, RT-qPCR and western blotting were conducted to examine the expression of epithelial and mesenchymal markers in HEC-1A cells transfected with control miR or pre-miR-21-5p. The results demonstrated that E-cadherin was significantly decreased, whereas fibronectin, $\mathrm{N}$-cadherin and vimentin were increased by pre-miR-21-5p in HEC-1A cells (Fig. 2D and $\mathrm{E})$. In addition, the MTT assay was used to determine whether miR-21-5p could affect proliferation of HEC-1A cells; overexpression of miR-21-5p promoted proliferation of HEC-1A cells (Fig. 2F).
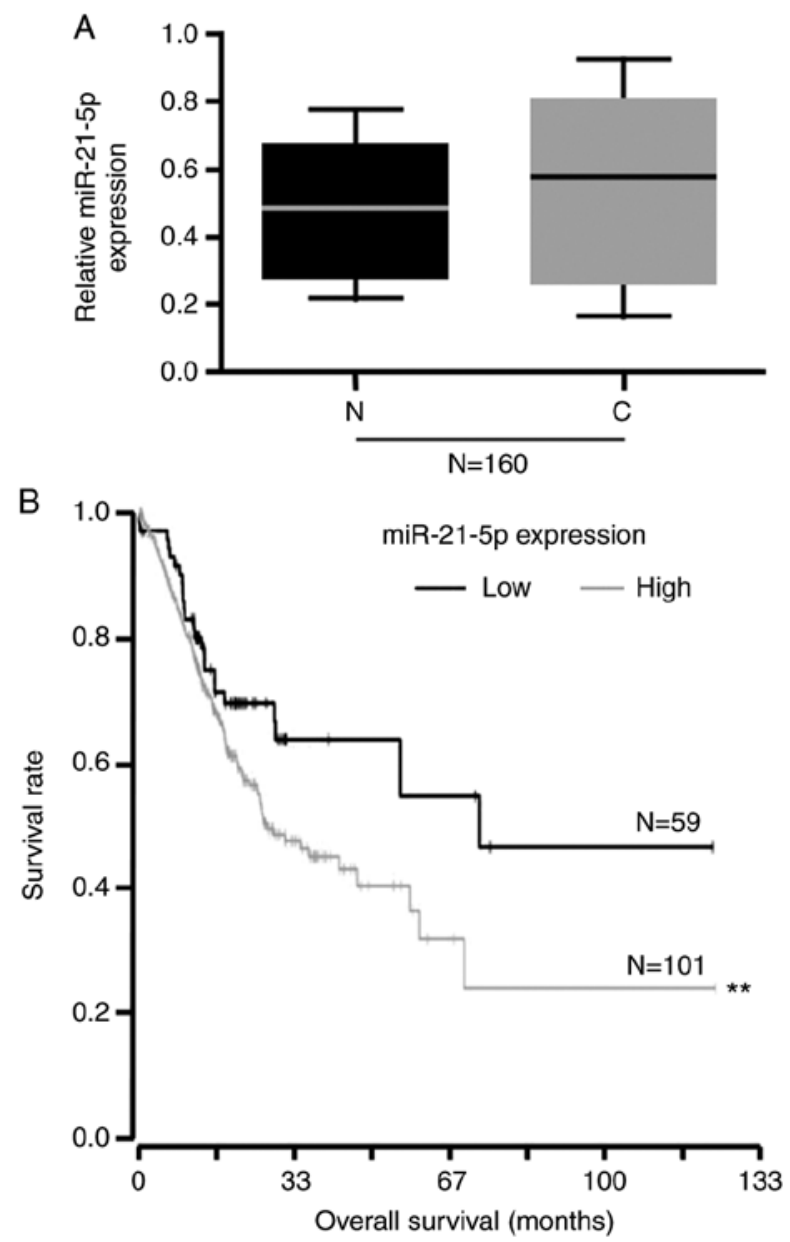

Figure 1. Association between miR-21-5p expression and overall survival. (A) Reverse transcription-quantitative polymerase chain reaction analysis of miR-21-5p in 160 endometrial cancer tissues and adjacent normal tissues. (B) Overall survival of patients with endometrial cancer was calculated by the Kaplan-Meier method and compared using the log-rank test, according to miR-21-5p expression. Each drop in the survival curve represents the time of $\geq 1$ cases of patient mortality. The vertical lines represent the time of censoring. ${ }^{* *} \mathrm{P}<0.01$. C, cancer tissues; miR-21-5p, microRNA-21-5p; $\mathrm{N}$, normal tissues.

miR-21-5p knockdown promotes mesenchymal to epithelial transition (MET) in endometrial cancer cell lines. The present study also investigated the effects of an inhibitor of miR-21-5p, anti-miR-21-5p. A total of $48 \mathrm{~h}$ post-transfection, miR-21-5p expression was determined by RT-qPCR; miR-21-5p expression was downregulated by anti-miR-21-5p in AN3CA cells (Fig. 3A). Silencing miR-21-5p promoted visible alterations in AN3CA cell morphology (Fig. 3B). To further study the role of anti-miR-21-5p in MET, RT-qPCR and western blotting were used to examine the expression levels of epithelial and mesenchymal markers in AN3CA cells transfected with anti-miR-21-5p or a scramble miR control.E-cadherin expression was increased, whereas fibronectin, $\mathrm{N}$-cadherin and vimentin expression were decreased by anti-miR-21-5p (Fig. 3C and D). The MTT assay was used to determine whether anti-miR-21-5p could affect proliferation of AN3CA cells; silencing miR-21-5p inhibited proliferation of AN3CA cells (Fig. 3E).

miR-21-5p regulates SOX17 expression in endometrial cancer. To screen target genes of miR-21-5p, the online software 
A

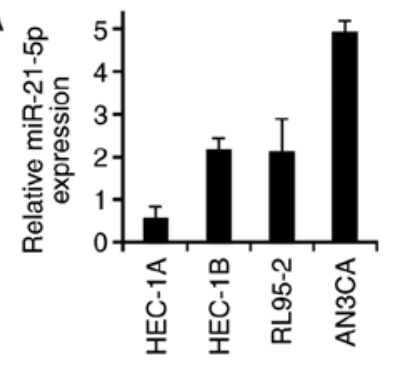

C

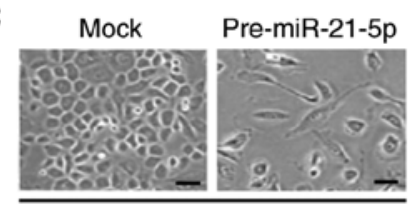

HEC-1A

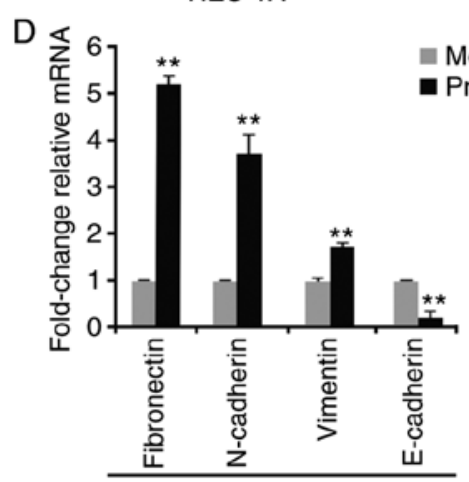

HEC-1A

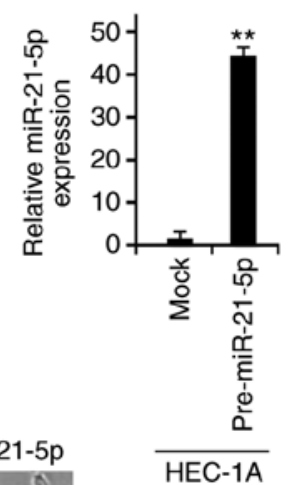

Mock

Pre-miR-21-5p
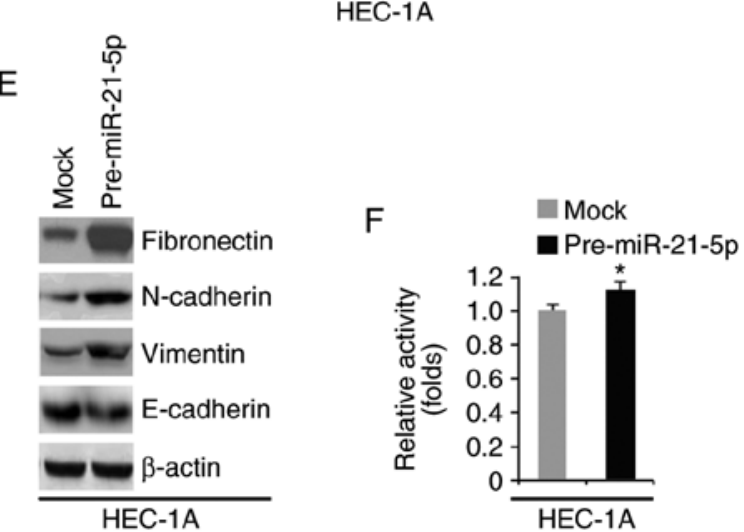

Figure 2. miR-21-5p promotes epithelial to mesenchymal transition in endometrial cancer. (A) RT-qPCR analysis of miR-21-5p in HEC-1A, HEC-1B, AN3CA and RL95-2 cells. (B) RT-qPCR analysis of miR-21-5p in HEC-1A cells transfected as indicated $(n=3)$. (C) HEC-1A cells were transfected as indicated, and images of the cells were captured. Scale bars, $50 \mu \mathrm{m} \mathrm{n}=3$. (D) RT-qPCR analysis of fibronectin, $\mathrm{N}$-cadherin, vimentin and E-cadherin in HEC-1A cells transfected as indicated $(n=3)$. (E) Western blot analysis of fibronectin, $\mathrm{N}$-cadherin, vimentin and E-cadherin in HEC-1A cells transfected as indicated $(n=3)$. (F) MTT assay of HEC-1A cells transfected as indicated $(n=3) .{ }^{*} \mathrm{P}<0.05$ and ${ }^{* * *} \mathrm{P}<0.01$ vs. the Mock group. miR-21-5p, microRNA-21-5p; RT-qPCR, reverse transcription-quantitative polymerase chain reaction.

miRanda (http://www.microrna.org/microrna/home.do) was used. A large number of target genes were identified, including SOX17. Recently, SOX17 has been reported to act as a tumor suppressor gene in endometrial cancer (8). Therefore, the present study focused on this gene. miR-21-5p target sites were detected on the 3'-untranslated region (3'UTR) of SOX17, and identical sequences were revealed among different species (Fig. 4A).
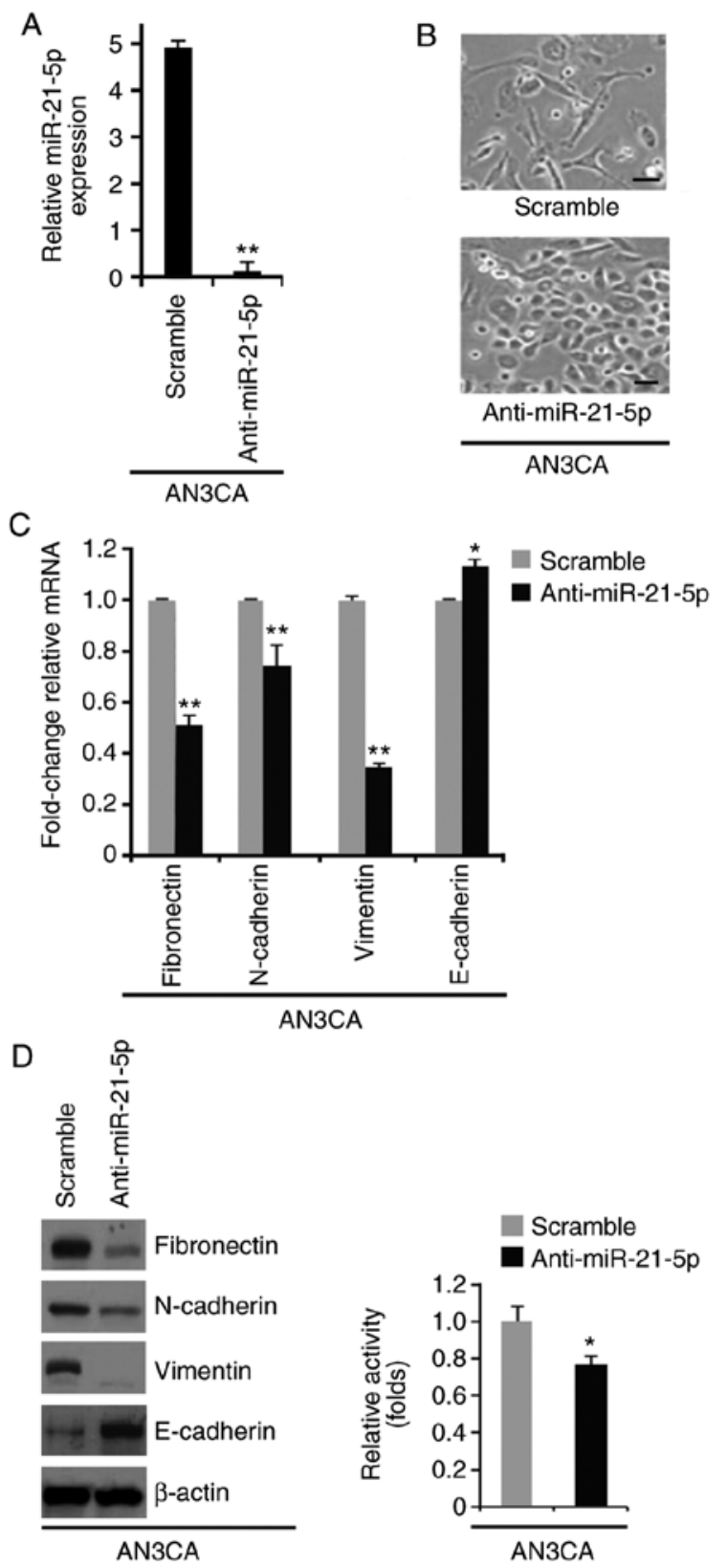

Figure 3. Silencing of miR-21-5p promotes mesenchymal to epithelial transition in endometrial cancer. (A) RT-qPCR analysis of miR-21-5p in AN3CA cells transfected as indicated $(n=3)$. (B) AN3CA cells were transfected as indicated, and images of the cells were captured. Scale bars, $50 \mu \mathrm{m} \mathrm{n}=3$. (C) RT-qPCR analysis of fibronectin, N-cadherin, vimentin and E-cadherin in AN3CA cells transfected as indicated $(n=3)$. (D) Western blot analysis of fibronectin, N-cadherin, vimentin and E-cadherin in AN3CA cells transfected as indicated ( $n=3)$. (D) MTT assay of AN3CA cells transfected as indicated $(\mathrm{n}=3) .{ }^{*} \mathrm{P}<0.05$ and ${ }^{* *} \mathrm{P}<0.01$ vs. the Scramble group. miR-21-5p, microRNA-21-5p; RT-qPCR, reverse transcription-quantitative polymerase chain reaction.

To identify SOX17 expression in endometrial cancer cell lines, western blotting was performed. The results demonstrated that SOX17 protein expression was highest in HEC-1A cells and lowest in AN3CA cells (Fig. 4B). To determine the role of miR-21-5p in regulating SOX17 expression, RT-qPCR was conducted to examine SOX17 mRNA expression in HEC-1A cells transfected with control miR (mock) or pre-miR-21-5p. The results demonstrated that pre-miR-21-5p inhibited SOX17 mRNA expression in HEC-1A cells (Fig. 4C). To investigate 
A
B

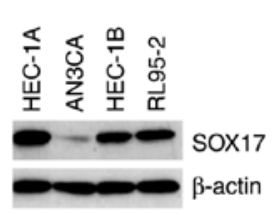

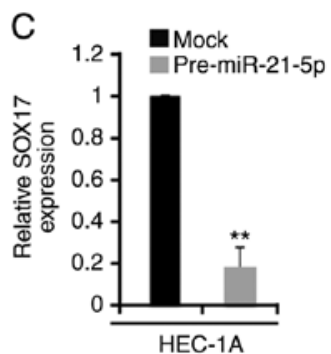

$\mathrm{F}$
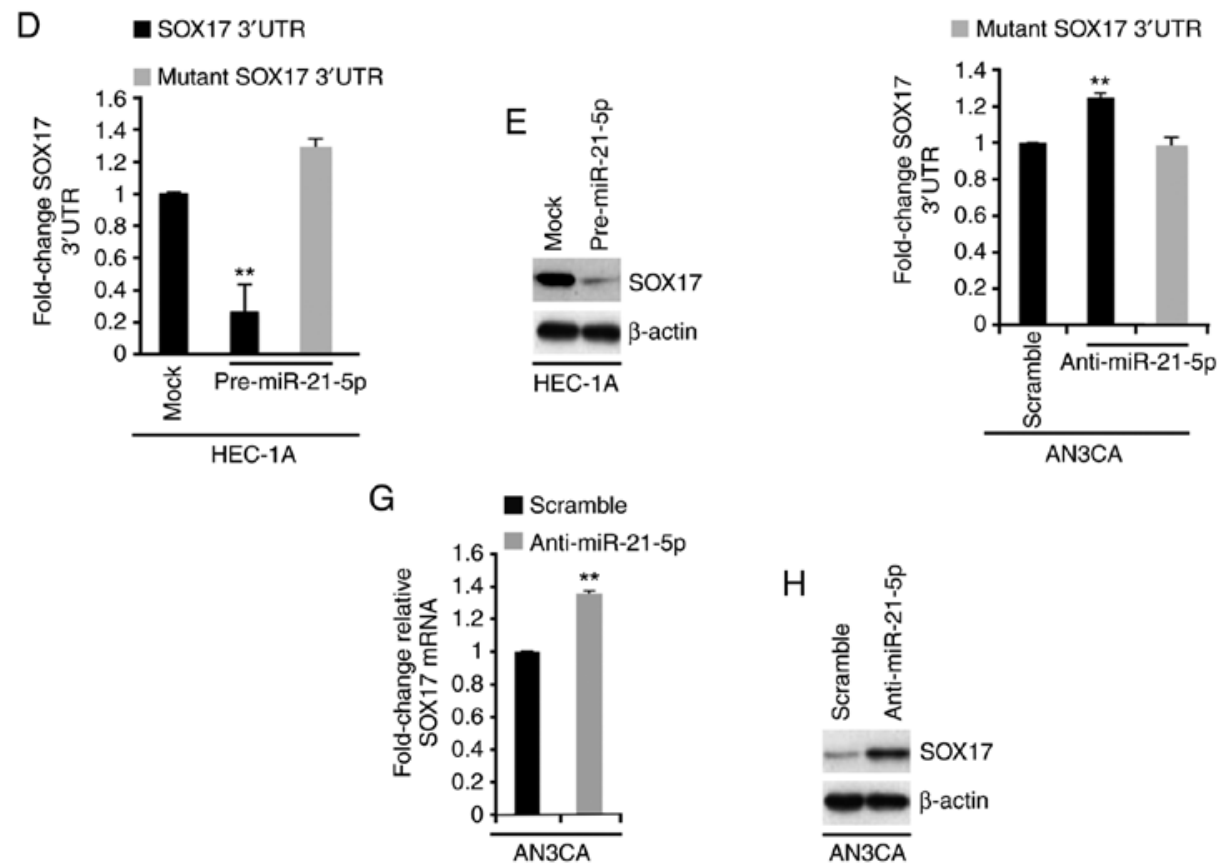

Figure 4. miR-21-5p regulates SOX17 expression in endometrial cancer. (A) Predicted binding sites of miR-21-5p and SOX17, as determined using the miRanda algorithm. Conservation of miR-21-5p targeting sites in the 3'UTR of SOX17 (WT), and the MUT sequence that abrogates miR-21-5p binding to SOX17 mRNA. (B) Western blotting of SOX17 in HEC-1A, AN3CA, HEC-1B and RL95-2 cells ( $\mathrm{n}=3$ ). (C) RT-qPCR analysis of SOX17 in HEC-1A cells transfected as indicated ( $\mathrm{n}=3$ ). ${ }^{* *} \mathrm{P}<0.01$ vs. the Mock group. (D) Luciferase reporter assay in HEC-1A cells transfected as indicated ( $\mathrm{n}=3$ ). ${ }^{* *} \mathrm{P}<0.01 \mathrm{vs}$. mutant SOX17 $3^{\prime} \mathrm{UTR}$. (E) Western blot analysis of SOX17 in HEC-1A cells transfected as indicated $(n=3)$. (F) Luciferase reporter assay in AN3CA cells transfected as indicated $(\mathrm{n}=3)$. (G) RT-qPCR analysis of SOX17 mRNA in AN3CA cells transfected as indicated $(\mathrm{n}=3)$. (H) Western blot analysis of SOX17 protein in AN3CA cells transfected as indicated $(\mathrm{n}=3) .{ }^{* *} \mathrm{P}<0.01$ vs. the Scramble group. 3'UTR, 3'-untranslated region; miR-21-5p, microRNA-21-5p; MUT, mutant; RT-qPCR, reverse transcription-quantitative polymerase chain reaction; SOX17, SRY-box 17; WT, wild type.

the direct regulation of SOX17 by miR-21-5p, a luciferase reporter assay was performed. Wild type SOX17 3'UTR was introduced into HEC-1A cells. The results revealed that the luciferase activity of wild type plasmids was suppressed by pre-miR-21-5p (Fig. 4D). Subsequently, three bases in the predicted sites were mutated (Fig. 4A), and mutant SOX17 3'UTR was introduced into HEC-1A cells. As expected, the luciferase activity of mutant reporters was not be affected by miR-21-5p in HEC-1A cells (Fig. 4D). In addition, western blotting was conducted to determine SOX17 protein expression in HEC-1A cells transfected with control miR or pre-miR-21-5p; SOX17 protein expression was also decreased by pre-miR-21-5p (Fig. 4E).

The present study also investigated whether silencing miR-21-5p could regulate SOX17 expression in AN3CA cells. AN3CA cells were transfected with anti-miR-21-5p or a scramble miR control. A total of $48 \mathrm{~h}$ post-transfection, miR-21-5p expression was determined by RT-qPCR, and miR-21-5p

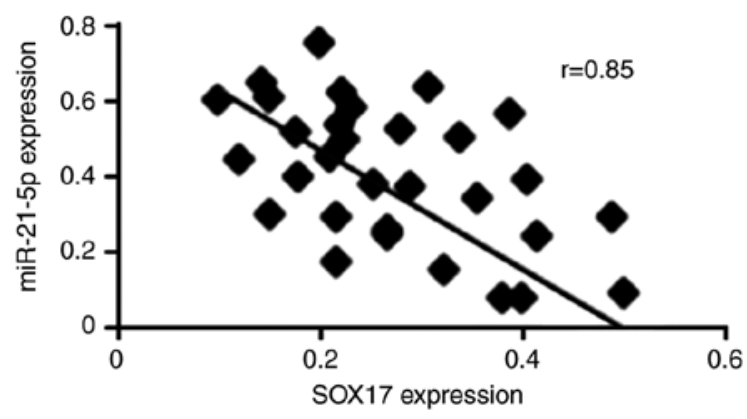

Figure 5. SOX17 is inversely correlated with miR-21-5p expression in endometrial cancer. Spearman correlation for SOX17 mRNA and miR-21-5p in endometrial cancer $(\mathrm{n}=30)$. miR-21-5p, microRNA-21-5p; SOX17, SRY-box 17.

expression was decreased by anti-miR-21-5p (Fig. 3A). A luciferase reporter assay was subsequently performed to examine 
Table II. Association between SOX17 expression and pathological parameters of endometrial cancer.

SOX17 expression

\begin{tabular}{lrr}
\cline { 3 - 3 } Clinical parameter & Low $(\%)$ & High (\%) \\
\hline Lymphatic metastasis & $93(51)$ & $44(49)$ \\
No & $16(50)$ & $7(50)$ \\
Yes & & \\
Invasion depth & $72(68)$ & $34(32)$ \\
$<1 / 2$ & $27(68)$ & $12(32)$ \\
$>1 / 2$ & $10(67)$ & $5(33)$ \\
Limited to endometrium & & 0.12 \\
\hline
\end{tabular}

P-values were calculated using $\chi^{2}$ tests for categorical variables. Percentages show the ratios of patients with high SOX17 or low SOX17 expression for each item. SOX17, SRY-box 17.

whether anti-miR-21-5p could regulate the activity of SOX17 3'UTR in AN3CA cells. The results demonstrated that silencing miR-21-5p promoted SOX17 3'UTR activity in AN3CA cells (Fig. 4F). RT-qPCR and western blotting were used to examine the mRNA and protein expression levels of SOX17 in AN3CA cells transfected with a scramble miR control and anti-miR-21-5p. Silencing miR-21-5p promoted SOX17 mRNA and protein expression (Fig. 4G and $\mathrm{H}$ ).

SOX17 expression is negatively correlated with miR-21-5p expression in endometrial cancer. To investigate the association between SOX17 mRNA expression and miR-21-5p expression in endometrial cancer, RT-qPCR was used to examine SOX17 and miR-21-5p expression in 30 endometrial cancer tissues [there is an $80 \%$ power to detect a moderate $(r \geq 0.4)$ correlation between SOX17 and miR-21-5p expression, when sample size is 30]. The results demonstrated that SOX17 mRNA was negatively correlated with miR-21-5p expression $(\mathrm{r}=0.85, \mathrm{P}<0.01$; Fig. 5).

SOX17 regulates EMT in endometrial cancer cell lines. To determine the role of SOX17 in endometrial cancer, AN3CA cells were transfected with SOX17-expressing plasmids or empty vectors (mock). SOX17 protein was significantly increased by SOX17-expressing plasmids (Fig. 6A). The increase in SOX17 expression induced visible alterations in AN3CA cell morphology (Fig. 6B). To confirm that these visible alterations in cell morphology were associated with EMT, western blot analysis was conducted to determine the expression levels of epithelial and mesenchymal markers in AN3CA cells transfected with SOX17-expressing plasmids or empty vectors. E-cadherin was markedly increased, whereas fibronectin, N-cadherin and vimentin were decreased by SOX17 (Fig. 6C). Furthermore, SOX17 overexpression inhibited proliferation of AN3CA cells (Fig. 6D). The present study also determined the effects of an inhibitor of SOX17, shSOX17. A total of $48 \mathrm{~h}$ post-transfection, SOX17 expression was examined by western blotting. shSOX17 inhibited SOX17 protein expression in HEC-1A cells (Fig. 6E) and silencing SOX17 promoted visible alterations in HEC-1A cell morphology (Fig. 6F). To analyze the role of SOX17 in EMT, western blotting was performed to determine the expression levels of epithelial and mesenchymal markers in HEC-1A cells transfected with shSOX17 or a scramble control. E-cadherin was decreased, whereas fibronectin, N-cadherin and vimentin were increased by shSOX17 in the cells (Fig. 6G). Cell proliferation was determined by MTT assay; silencing SOX17 promoted proliferation of HEC-1A cells (Fig. 6H).

Association between SOX17 expression and clinicopathological features in gastric cancer. The association between SOX17 expression and clinicopathological features was summarized in Table II. The results demonstrated that SOX17 expression was not associated with lymphatic metastasis $(\mathrm{P}>0.05)$ and invasion depth $(\mathrm{P}>0.05)$.

Association between SOX17 expression and overall survival. To identify SOX17 expression in endometrial cancer tissues, immunohistochemistry was performed. Kaplan-Meier curves were applied to assess the overall survival for 160 patients with primary endometrial cancers, stratified based on tumor SOX17 expression. Representative images of immunohistochemistry staining are shown in Fig. 7A. There was a significant difference between the two overall survival curves; survival among patients with low-level SOX17 expression was much poorer than survival among patients with high-level SOX17 expression (Fig. 7B).

\section{Discussion}

The primary aim of the present study was to investigate the role of miR-21-5p and SOX17 in endometrial cancer. This study is the first, to the best of our knowledge, to demonstrate that miR-21-5p may promote EMT by targeting SOX17 and is correlated with poor survival in patients with endometrial cancer.

The present study revealed that miR-21-5p expression was highest in AN3CA cells and lowest in HEC-1A cells. HEC-1A cells are derived from moderately differentiated endometrial cancer, whereas AN3CA cells are derived from undifferentiated endometrial cancer (26). Therefore, miR-21-5p may be 
A

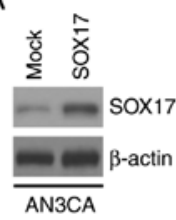

$\mathrm{E}$

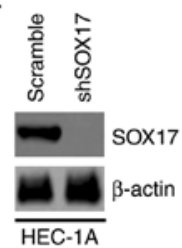

B

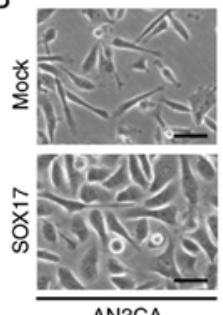

$\mathrm{F}$

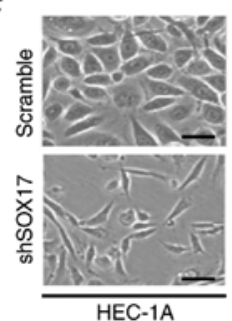

C

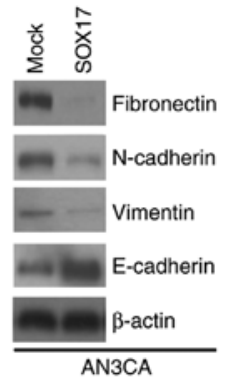

G

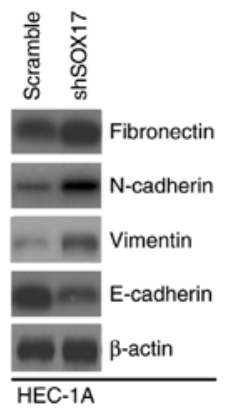

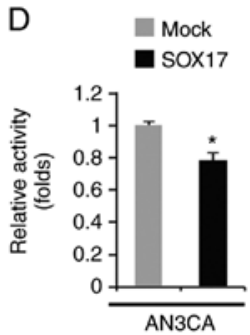

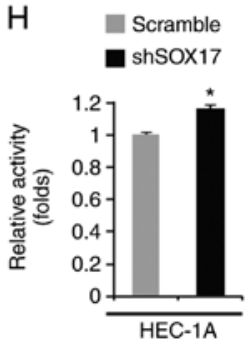

Figure 6. SOX17 regulates EMT in endometrial cancer. (A) Western blot analysis of SOX17 in AN3CA cells transfected as indicated (n=3). (B) AN3CA cells were transfected as indicated, and images of the cells were captured. Scale bars, $50 \mu \mathrm{m}$. $\mathrm{n}=3$. (C) Western blot analysis of fibronectin, $\mathrm{N}$-cadherin, vimentin and E-cadherin in AN3CA cells transfected as indicated $(n=3)$. (D) MTT assay of AN3CA cells transfected as indicated $(n=3)$. " $P<0.05$ vs. the Mock group. (E) Western blot analysis of SOX17 in HEC-1A cells transfected as indicated (n=3). (F) HEC-1A cells were transfected as indicated, and images of the cells were captured. Scale bars, $50 \mu \mathrm{m} . \mathrm{n}=3$. (G) Western blot analysis of fibronectin, $\mathrm{N}$-cadherin, vimentin and E-cadherin in HEC-1A cells transfected as indicated $(\mathrm{n}=3)$. (H) MTT assay of HEC-1A cells transfected as indicated $(\mathrm{n}=3)$. ${ }^{*} \mathrm{P}<0.05$ vs. the Scramble group. miR-21-5p, microRNA-21-5p; shSOX17, short hairpin RNA-SOX17; SOX17, SRY-box 17.

A
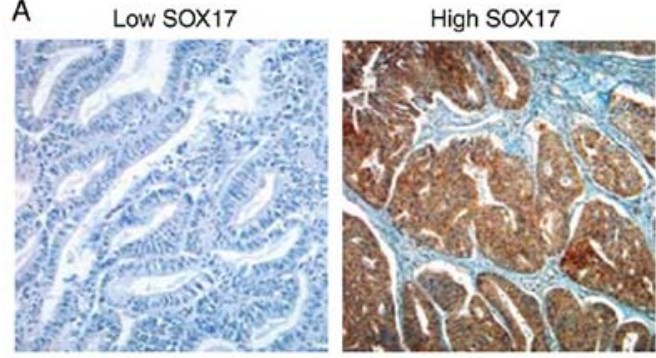

B

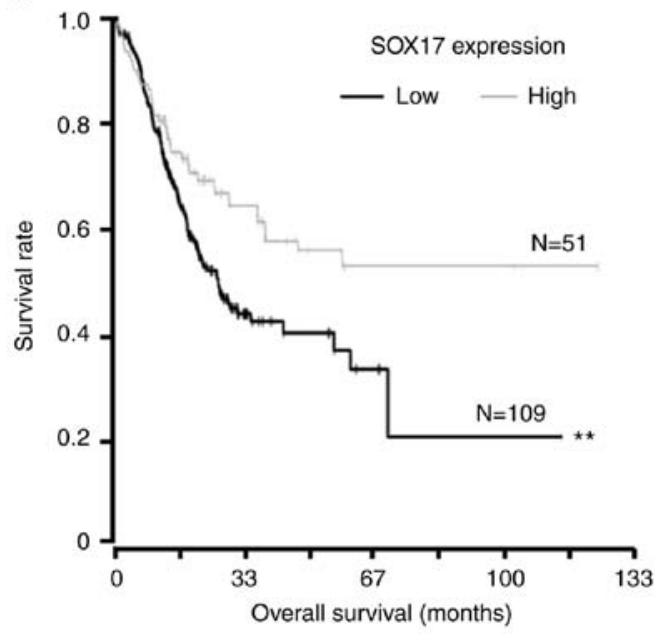

Figure 7. Association between SOX17 protein expression and overall survival. (A) Representative images of immunohistochemical staining of low SOX17 protein expression and high SOX17 protein expression. (B) Overall survival of 160 patients with primary endometrial cancer, stratified based on the expression of tumor SOX17. Each drop in the survival curve represents the time of $\geq 1$ cases of patient mortality. The vertical lines represent the time of censoring. P-value was calculated using the log rank test. ${ }^{* *} \mathrm{P}<0.01$. SOX17, SRY-box 17. associated with poor differentiation. EMT is a critical developmental program whereby epithelial cells obtain mesenchymal characters $(27,28)$, and it is a crucial step during endometrial cancer metastasis $(27,28)$. The present study revealed that miR-21-5p promoted EMT, whereas silencing miR-21-5p reversed EMT in endometrial cancer cells. miR-21-5p expression is dysregulated in endometrial cancer (15) and promotes the progression of endometrial cancer (17). Consistent with a previous report (17), the present study observed that miR-21-5p expression was inversely correlated with overall survival.

SOX17 protein expression was highest in HEC-1A cells and lowest in AN3CA cells, thus indicating that SOX17 protein expression may be associated with better differentiation. In a previous study, overexpression of SOX17 inhibited proliferation and promoted apoptosis of HEC-1B cells, and inhibited growth of endometrial cancer in animal models (8). Consistent with this previous report (8), the present results demonstrated that SOX17 inhibited proliferation of AN3CA cells. Furthermore, the present study is the first, to the best of our knowledge, to indicate that overexpression of SOX17 may inhibit EMT in endometrial cancer cell lines.

SOX17 expression is correlated with longer recurrence-free survival in endometrial cancer (8). The present study indicated that tumor SOX17 expression was correlated with improved overall survival in Chinese patients, which is in line with a previous report (8). These results suggested that SOX17 may be a candidate tumor suppressor gene in endometrial cancer.

In conclusion, SOX17 was revealed to function as a candidate tumor suppressor gene in the progression of endometrial cancer by regulating EMT and proliferation. Furthermore, the present study suggested that miR-21-5p may be a functional target for endometrial cancer therapy. 


\section{Acknowledgements}

Not applicable.

\section{Funding}

The present study was supported by Jinan Maternity and Child Health Care Hospital.

\section{Availability of data and materials}

The datasets used and/or analyzed during the current study are available from the corresponding author on reasonable request.

\section{Authors' contributions}

$\mathrm{CW}$ and YH performed the majority of the experimental work, initially conceived the study and wrote a draft of the manuscript. QL performed the remainder of the experimental work. All authors read and approved the final manuscript.

\section{Ethics approval and consent to participate}

The present study was approved by the ethics committee of Maternity and Child Health Care Hospital (Jinan, China), and each patient provided written informed consent at the time of enrollment.

\section{Patient consent for publication}

Consent for publication was obtained from each patient.

\section{Competing interests}

All authors declare that there are no competing interests.

\section{References}

1. Janda M, Gebski V, Davies LC, Forder P, Brand A, Hogg R, Jobling TW, Land R, Manolitsas T, Nascimento M, et al: Effect of total laparoscopic hysterectomy vs total abdominal hysterectomy on disease-free survival among women with stage I endometrial cancer: A randomized clinical trial. JAMA 317 1224-1233, 2017.

2. Amant F, Moerman P, Neven P, Timmerman D, Van Limbergen E and Vergote I: Endometrial cancer. Lancet 366: 491-505, 2005.

3. Kamachi Y and Kondoh H: Sox proteins: Regulators of cell fate specification and differentiation. Development 140: 4129-4144 2013.

4. Betel D, Wilson M, Gabow A, Marks DS and Sander C: The microRNA. org resource: Targets and expression. Nucleic Acids Res 36: D149-D153, 2008.

5. Ye YW, Wu JH, Wang CM, Zhou Y, Du CY, Zheng BQ, Cao X, Zhou XY, Sun MH and Shi YQ: Sox17 regulates proliferation and cell cycle during gastric cancer progression. Cancer Lett 307: 124-131, 2011.

6. Yin D, Jia Y, Yu Y, Brock MV, Herman JG, Han C, Su X, Liu Y and Guo M: SOX17 methylation inhibits its antagonism of Wnt signaling pathway in lung cancer. Discov Med 14: 33-40, 2012

7. Jia Y, Yang Y, Zhan Q, Brock MV, Zheng X, Yu Y, Herman JG and Guo M: Inhibition of SOX17 by microRNA 141 and methylation activates the WNT signaling pathway in esophageal cancer. J Mol Diagn 14: 577-585, 2012.
8. Zhang Y, Bao W, Wang K, Lu W, Wang H, Tong H and Wan X: SOX17 is a tumor suppressor in endometrial cancer. Oncotarget 7: 76036-76046, 2016.

9. Kuo I, Wu CC, Chang JM, Huang YL, Lin CH, Yan JJ, Sheu BS, Lu PJ, Chang WL, Lai WW and Wang YC: Low SOX17 expression is a prognostic factor and drives transcriptional dysregulation and esophageal cancer progression. Int J Cancer 135: 563-573, 2014.

10. Zhang Y, Jiang F, Bao W, Zhang H, He X, Wang H and Wan X SOX17 increases the cisplatin sensitivity of an endometrial cancer cell line. Cancer Cell Int 16: 29, 2016.

11. Vonlanthen S, Heighway J, Altermatt H, Gugger M, Kappeler A, Borner MM, van Lohuizen M and Betticher DC: The bmi-1 oncoprotein is differentially expressed in non-small cell lung cancer and correlates with INK4A-ARF locus expression. Br J Cancer 84: 1372-1376, 2001.

12. Lu J, Getz G, Miska EA, Alvarez-Saavedra E, Lamb J, Peck D, Sweet-Cordero A, Ebert BL, Mak RH, Ferrando AA, et al: MicroRNA expression profiles classify human cancers. Nature 435: 834-838, 2005.

13. Browne G, Taipaleenmäki H, Stein GS, Stein JL and Lian JB: MicroRNAs in the control of metastatic bone disease. Trends Endocrinol Metab 25: 320-327, 2014.

14. Mei Z, Zhou L, Zhu Y, Jie K, Fan D, Chen J, Liu X, Jiang L, Jia $\mathrm{Q}$ and $\mathrm{Li} \mathrm{W}$ : Interleukin-22 promotes papillary thyroid cancer cell migration and invasion through microRNA-595/Sox17 axis. Tumour Biol 37: 11753-11762, 2016.

15. Myatt SS, Wang J, Monteiro LJ, Christian M, Ho KK, Fusi L, Dina RE, Brosens JJ, Ghaem-Maghami S and Lam EW: Definition of microRNAs that repress expression of the tumor suppressor gene FOXO1 in endometrial cancer. Cancer Res 70: 367-377, 2010.

16. Dong P, Kaneuchi M, Watari H, Hamada J, Sudo S, Ju J and Sakuragi N: MicroRNA-194 inhibits epithelial to mesenchymal transition of endometrial cancer cells by targeting oncogene BMI-1. Mol Cancer 10: 99, 2011.

17. Qin X, Yan L, Zhao X, Li C and Fu Y: MicroRNA-21 overexpression contributes to cell proliferation by targeting PTEN in endometrioid endometrial cancer. Oncol Lett 4: 1290-1296, 2012.

18. Liao XH,Lu DL, Wang N,Liu LY, Wang Y,Li YQ, Yan TB, Sun XG, $\mathrm{Hu} P$ and Zhang TC: Estrogen receptor $\alpha$ mediates proliferation of breast cancer MCF-7 cells via a p21/PCNA/E2F1-dependent pathway. FEBS J 281: 927-942, 2014.

19. Wang P, Zhang L, Zhang J and Xu G: MicroRNA-124-3p inhibits cell growth and metastasis in cervical cancer by targeting IGF2BP1. Exp Ther Med 15: 1385-1393, 2018.

20. Livak KJ and Schmittgen TD: Analysis of relative gene expression data using real-time quantitative PCR and the 2(-Delta Delta C(T)) method. Methods 25: 402-408, 2001.

21. Junn E, Lee KW, Jeong BS, Chan TW, Im JY and Mouradian MM: Repression of alpha-synuclein expression and toxicity by microRNA-7. Proc Natl Acad Sci USA 106: 13052-13057, 2009.

22. Zhang SY, Caamano J, Cooper F, Guo X and Klein-Szanto AJ: Immunohistochemistry of cyclin D1 in human breast cancer. Am J Clin Pathol 102: 695-698, 1994.

23. Hauke J and Kossowski T: Comparison of values of Pearson's and Spearman's correlation coefficients on the same sets of data. Quaestiones Geographicae 30: 87-93, 2011.

24. Metz CE: Basic principles of ROC analysis. Semin Nucl Med 8: 283-298, 1978.

25. Zweig MH and Campbell G: Receiver-operating characteristic (ROC) plots: A fundamental evaluation tool in clinical medicine. Clin Chem 39: 561-577, 1993.

26. Gao J, Niwa K, Sun W, Takemura M, Lian Z, Onogi K, Seishima M, Mori $\mathrm{H}$ and Tamaya $\mathrm{T}$ : Non-steroidal anti-inflammatory drugs inhibit cellular proliferation and upregulate cyclooxygenase-2 protein expression in endometrial cancer cells. Cancer Sci 95: 901-907, 2004.

27. Tsuji T, Ibaragi S and Hu GF: Epithelial-mesenchymal transition and cell cooperativity in metastasis. Cancer Res 69: 7135-7139, 2009.

28. Gavert N and Ben-Ze'ev A: Epithelial-mesenchymal transition and the invasive potential of tumors. Trends Mol Med 14: 199-209, 2008.

This work is licensed under a Creative Commons Attribution-NonCommercial-NoDerivatives 4.0 International (CC BY-NC-ND 4.0) License. 